\title{
Elisabeth Schulze-Busacker, Vittorio Fortunati (dir.), Par les siècles et par les genres. Mélanges en l'honneur de Giorgetto Giorgi
}

\section{Alessio Mattana}

\section{(2) OpenEdition}

\section{Journals}

\section{Edizione digitale}

URL: http://journals.openedition.org/studifrancesi/1505

DOI: 10.4000/studifrancesi. 1505

ISSN: 2421-5856

\section{Editore}

Rosenberg \& Sellier

\section{Edizione cartacea}

Data di pubblicazione: 1 décembre 2015

Paginazione: 648-649

ISSN: 0039-2944

\section{Notizia bibliografica digitale}

Alessio Mattana, «Elisabeth Schulze-Busacker, Vittorio Fortunati (dir.), Par les siècles et par les genres. Mélanges en I'honneur de Giorgetto Giorgi», Studi Francesi [Online], 177 (LIX | III) | 2015, online dal 01 décembre 2015, consultato il 10 janvier 2021. URL: http://journals.openedition.org/studifrancesi/1505 ; DOI: https://doi.org/10.4000/studifrancesi.1505

Questo documento è stato generato automaticamente il 10 janvier 2021.

\section{cc) (†) $\odot$}

Studi Francesi è distribuita con Licenza Creative Commons Attribuzione - Non commerciale - Non opere derivate 4.0 Internazionale. 


\title{
Elisabeth Schulze-Busacker, Vittorio Fortunati (dir.), Par les siècles et par les genres. Mélanges en l'honneur de Giorgetto Giorgi
}

\author{
Alessio Mattana
}

\section{NOTIZIA}

ELISABETH SCHULZE-BUSACKER, VITTORIO FORTUNATI (dir.), Par les siècles et par les genres.

Mélanges en l'honneur de Giorgetto Giorgi, Paris, Classiques Garnier, 2014, pp. 830.

1 Per la cura di Elisabeth SCHULZE-BUSACKER e di Vittorio FORTUNATI, convergono in questo volume - nato dal desiderio di colleghi e amici di una vita di tributare onore alla poliedrica attività di Giorgetto Giorgi - quarantotto interventi che si propongono di restituire le cadenze di una voce culturale ricca di autorità non solo nella francesistica, disciplina nella quale Giorgi è stato fin da molto presto e continua ad essere centrale, ma della letteratura tout court. I curatori di questo settantottesimo volume della collana Rencontres presentano il bouquet di articoli redatti da una serie di specialisti (per la quasi totalità italiani e francesi) in ordine alfabetico, optando per una brevissima presentazione in cui la diversità dei contributi viene intesa come «écho aux vastes intérêts de notre ami pour la littérature française des derniers siècles». La curatela si muove agilmente lungo i due assi proposti nel titolo, quello cronologico e quello di genere: per orientarsi lungo i quarantotto contributi la divisione per secoli sembra essere la scelta più interessante al fine di apprezzare la distribuzione, l'eterogeneità e le inaspettate comunanze tematiche tra gli interventi.

2 L'unico contributo di orbita non-letteraria stricto sensu è quello di Annalisa BAICCHI (pp. 23-50), scritto in inglese, che discute di modelli cognitivi e speech acts. Il resto degli interventi si rivolge alla letteratura, oscillando tra close-reading e analisi socio- 
culturale, in due casi senza vincoli temporali: Dario GIBELLI (pp. 349-368) analizza la dissimulazione operata da testi che mettono in crisi le massime conversazionali di Grice; Jacqueline RISSET (pp. 603-616) rintraccia il mito dell'Odissea in, tra gli altri, du Bellay, Ronsard, Montaigne, Fénelon, Marivaux e Blanchot.

L'epoca più lontana analizzata è il primo Umanesimo francese, grazie a Dario CECCHETTI (pp. 165-188) che ci guida nella ricca poesia in latino di Jean Gerson, Nicolas de Clamanges e Laurent de Premierfait; subito successivo è il contributo di Anna Maria FINOLI (pp. 301-314) che descrive il colto ambiente dei Borgogna attraverso due biographies chevaleresques della biblioteca di Filippo II. Il Cinquecento per Nerina CLERICI BALMAS (pp. 189-193) è riscoperta delle parole enigmatiche di Salomé in un mystère; Daniela MAURI (pp. 435-455), invece, ne enfatizza l'eclettismo di taglio rinascimentale restituendo il «carattere multiforme» della grotta nei romanzi di Béroalde de Verville. Il fulcro cronologico del volume è, come detto, il Seicento. Si discute molto dell'influenza greco-latina: con Emmanuel BURY in relazione a Madeleine de Scudéry (pp. 103-115); con Francesco FIORENTINo (pp. 315-322) e quel Cinna di Corneille uscito a ridosso di due congiure contro Richelieu (1641-42); con Marie-Gabrielle LALLEMAND (pp. 383-397), contributo innestato sull'analisi di Giorgi circa l'influenza delle Éthiopiques di Eliodoro sulle forme lunghe; con Benedetta PAPASOGLI (pp. 525-536), che, scrivendo di Mnemosine, scopre dei «restes de platonisme» in d'Urfé e de Sales; con Philippe SELLIER (pp. 723-733) che legge Les Aventures de Télémaque attraverso la "grille d'analyse» derivata da Boileau, Rapin, Le Bossu, Bernard Lamy. Ma vi sono, inoltre, le rifrazioni epistolari tra «récit de fiction» e «fiction du récit» dell'intervento di Magda CAMPANINI (pp. 117-133); il triangolo tra realtà, finzione e rappresentazione tracciato da Alberto CASTOLDI (pp. 153-163) a partire dalla Carte de Tendre; le responsabilità etiche (nella fattispecie di Fedra nell'Hypolite di Gilbert) analizzate da Daniela DALla VALLE (pp. 233-244); la creazione romanzesca in Les États et Empires de la Lune et du Soleil con Patrick DANDREY (pp. 245-257); la relazione tra tradizioni culturali francese e italiana nell'Astrée con Delphine DENIS (pp. 273-283); i tre trattati sul roman del Du Plaisir del 1683 con Camille ESMEIN-SARRAZIN (pp. 285-300). Nello scandagliare questo secolo si esplorano anche l'innestarsi di missive nel Roman comique di Scarron (Vittorio FORTUNATI, pp. 323-332), le dinamiche di ricezione e i lettori ideali ai tempi di La Calprenède, Mme de Sévigné e Du Plaisir (Philippe HOURCADE, pp. 369-381), le strategie di legittimazione letteraria per generi come il conte de fées (Jole MORGANTE, pp. 471-490), le componenti antropologiche che Paul PELCKMANS individua in La Duchesse d'Estramène (pp. 537-551), le commistioni di «littérature légère et élégante» e "prose et [...] vers» in Le commerce galant tracciate da Franco PIVA (pp. 553-570); il rapporto tra fonti storiografiche e inventio autoriale e il confronto con il realismo stendhaliano intavolato da Giorgio SALE nel Dom Carlos di Saint-Réal (pp. 647-662). Al criticamente trascurato genere degli ana, infine, si dedica Francine wiLD (pp. 771-785).

Il Settecento è rappresentato in maniera più snella. François-Xavier cucHE ci parla de Les Trois Cousines di Dancourt (pp. 213-232), mentre Elisa BIANCARDI (pp. 51-72) confronta due trasposizioni italiane del Magasin des enfants di Madame Leprince de Beaumont. Benedetta CRAVERI (pp. 195-212), ritrae Louise-Honorine Crozat du Châtel tramite le sue splendide epistole e Letizia NORCI CAGIANO (pp. 511-524) situa il suo contributo tra i Guaranì, a partire dal celebre apologo di Montesquieu, abbracciando e l'utopia e la ricerca di modelli concreti. 
Un trittico di articoli guida la transizione verso il diciannovesimo secolo: Carminella BIONDI (pp. 73-88) affronta le ragioni storico-culturali della «figure du mulâtre»; Michel DELON (pp. 259-272) tocca l'immaginazione sotterranea tra geologia e letteratura, da Diderot a Stendhal. Lo spazio utopico (soprattutto pastorale) nella prosa tra 1790 e 1810 è l'argomento scelto da Jean-Marie Roulin (pp. 617-629) in quel clima che portò Chateaubriand ad affermare: "Tandis que la tragédie rougissait les rues, la bergerie florissait au théâtre». Il ritratto delle influenze su quest'ultimo centrale pensatore da parte delle Confessions di Rousseau è ciò che propone Aurelio PRINCIPATO (pp. 571-591), da un certo punto di vista parallelamente al compianto Lionello sozzi (pp. 735-749), che qui affrontava il problema dell'identità del poeta in autori italiani e francesi. Di Bien loin d'ici, sonetto unicum nelle Fleurs du mal, e di predecessori ed epigoni, leggiamo in Valerio MAGRELLI (pp. 419-433). Sugli stessi fiori si concentra Mario RICHTER (pp. 593-602), sceverando, nel linguaggio del poeta, «l'allusion symbolique [...] et l'ambiguïté (ou bisémie)». Sempre di poesia tratta il contributo di Alain NIDERST (pp. 491-494), dedicato a Fêtes galantes di Verlaine «à la fois baigné de nostalgie et destructeur de toute nostalgie». La bibliofilia e i rapporti editoriali tra Jouaust e Uzanne sono il soggetto di Maria Giulia LONGHI (pp. 399-417), mentre del piacere di lasciarsi catturare «dans les méandres et les arabesques de La Canne de jaspe» di Henri de Régnier discute Liana NISSIM (pp. 495-510); ancora, Giovanni Saverio SANTANGELO (pp. 663-704)ricostruisce l'influenza culturale di André Dacier sulla cultura europea di inizio Ottocento.

La sfera della contemporaneità è nutritamente rappresentata, tra contaminazioni e formazione culturale di Antonio Machado in Francia - con Giovanni CARAVAGGI (pp. 135-152) -, dimensione gnomica e ricezione in Raymond Radiguet - Stefano GENETTI (pp. 333-347) -, utilizzo della storia e scenografie milanesi in Giono - André-Alain MORELLo (pp. 457-469), - e scrittura del sé in biografie di individui non illustri-Gianfranco RUBINo (pp. 631-645). Fede che "guastò il piacere dei sensi», bellezza che rese «imperfetta la vocazione religiosa»: ecco le due polarità di cui Eleonora SPARVoLI (pp. 751-769) si serve per parlare del Léviathan di Julien Green. E, ancora, quel Parade del 1917 di Picasso, Satie, e Cocteau, e il ricordo di Cocteau su Apollinaire scoperto da Sergio zopPI (pp. 787-794) in un numero della rivista espressionista «Der Querschnitt» del 1922. A due scrittori tutt'ora in vita si dedicano, infine, Fabio sсотто (pp. 705-721) che ci parla dell'influenza della traduzione (Shakespeare, Petrarca) sui sonetti di Yves Bonnefoy - e Laura BRIGNOLI (pp. 89-102) che indaga sul rapporto tra scrittura del sé e autobiografia nel prismatico narratore di Écrire sur Tamara di Bénabou. 